\title{
Wirksamkeitsnachweis in der Phytotherapie
}

\author{
Reinhard Saller ${ }^{a, b}$ Jörg Melzer ${ }^{b, c}$ Günter Meng $^{d}$ Ursula von Mandach $^{\mathrm{e}}$ Karin Fürer \\ Herbert Schwabl ${ }^{f}$ Cecile Vennos ${ }^{f}$ \\ ${ }^{a}$ Institut für psychogene Erkrankungen, Zentrum für Gesundheit, Berlin, Deutschland, \\ ${ }^{\mathrm{b}}$ Institut für Komplementär- und integrative Medizin, Universitätsspital Zürich, Schweiz, \\ ' Institut für psychogene Erkrankungen, Zentrum für Gesundheit, Berlin, \\ ${ }^{d}$ Schwabe GmbH \& Co. KG, Karlsruhe, Deutschland, \\ ${ }^{\text {e }}$ Perinatale Pharmakologie, UniversitätsSpital Zürich, \\ ${ }^{\ddagger}$ Padma AG, Hinwil, Schweiz
}

Die moderne Phytotherapie ist im Rahmen ihrer materiellen Möglichkeiten ein durchaus forschungsaffiner Therapiebereich. Neben einem mehrdimensionalen Ansatz, anhand einer Heilpflanze beispielhaft ein potentielles phytotherapeutisches Therapiespektrum auszuloten geht es vor allem um eine reflektierte wissenschaftliche Auseinandersetzung mit Methodologie, Aussagekraft und notwendigen Modifizierungen von Metaanalysen sowie mit Ansätzen aus der Versorgungsforschung. Beide Vorgehensweisen bilden derzeit auch in der Phytotherapie einen viel beachteten Fokus der klinischen und klinisch-epidemiologischen Forschung. Die Metaanalysen beschäftigen sich naturgemäß mit bereits vorhandenen Studiendaten und treffen dabei jeweils eine begründete wenngleich selektive und forscherbezogen durchaus subjektive Auswahl. Spezifische phytotherapeutische Gesichtspunkte wie z.B. Besonderheiten von Extrakten oder Vergleichbarkeit von Phytotherapeutika werden bislang kaum wissenschaftlich angemessen be- rücksichtigt. In der Versorgungsforschung, die nicht durch fixe Studienvorgaben eingeschränkt ist, stehen Daten aus der flexiblen und variablen Praxis im Vordergrund. Dieser Ansatz kann es, bei allen Einschränkungen, ermöglichen, neue und therapierelevante Gesichtspunkte zu entdecken, z.B. im Hinblick auf Dosierungen. Der authentische Charakter von Phytotherapeutika als Vielstoffgemische in stofflicher und funktioneller Hinsicht mit genuinen pleiotropen Eigenschaften wird bislang in der Forschung, vor allem der pharmakologischen Forschung, nur selten adäquat aufgegriffen. Vergleichbar werden pflanzliche Arzneimittel noch immer präferentiell unter dem eher realitätsfernen Grundsatz gesehen: «one drug, one indication». Die Betrachtung und Beforschung von Phytotherapeutika als Netzwerkarzneimittel mit pleiotropen Signaturen weist auf zukunfsträchtige Vorgehensweisen hin.

Reinhard Saller

\section{KARGER}

(C) 2014 S. Karger GmbH, Freiburg

Fax +497614520714 


\section{Die Bedeutung der Metaanalysen in der Phytotherapie heute und morgen}

\author{
Autor: Jörg Melzer
}

\section{Einleitung}

In den letzten 20 Jahren erlangte das Konzept der evidenzbasierten Medizin (EbM) [1-4] im Medizinsystem den Status einer fast obligatorischen Methodik für die klinische Forschung. Dies lies von Anfang an Diskussionen unter Befürwortern und Kritikern aufkommen, unabhängig von ihrer Verortung in der damaligen Schulmedizin oder der sich universitär etablierenden Komplementärmedizin [5]. Obschon methodische Aspekte die Diskussion über die EbM dominieren (z.B. Publikations- oder Auswahlbias, Methoden bei Datenpooling oder Umgang mit fehlenden Daten) [6-10], finden sich unter anderem auch ökonomische (z.B. EbM zur medizinischen Qualitätssicherung vs. finanzieller Ressourcenallokation) $[1,5,11,12]$, ethische (z.B. Patientenpräferenz vs. Ökonomie, Asymmetrie Arzt-Patienten-Beziehung) [5, 11-13] und wissenschaftstheoretische (z.B. normative Methode, Paradigmenwechsel) [11,12,14] Argumentationen.

Nach über 35 Jahren der Anwendung der Methode der Metaanalyse (MA) $[8,9,15]$ und angesichts ihrer Bedeutung innerhalb der evidenzbasierten klinischen Forschung in der Komplementärmedizin der letzten 15-20 Jahre [1-4] stellt sich die Frage der kritischen Selbstreflektion, inwiefern MAs bei pflanzlichen Interventionen verbessert werden müssen. Weiterentwicklungen sind notwendig, um einen objektivierbaren wissenschaftlichen Diskurs weiterzuführen, der nicht in paradigmatischer Erstarrung [11-13] oder dem gegeneinander Ausspielen evidenz-, eminenz- oder epidensbasierter Positionen verharrt [3]. Methodische Weiterentwicklungen zu MA sind nicht neu: das zeigt einerseits die Entwicklung systematischer Reviews zu metanarrativen Reviews mit multidisziplinärer Perspektive mittels Kombination qualitativer und systematischer Methoden bei komplexen Fragestellungen [16], andererseits die Weiterentwicklung des allgemeinen CONSORT-Statements (Consolidated Standards of Reporting Trials) für klinische Studien [17] zum CONSORT-Statements für «pflanzliche Interventionen» [18], auch wenn es noch nicht ausreichend genutzt wird [19].

Die folgenden Ausführungen begrenzen sich auf ausgewählte methodische Aspekte von MA, die vor allem in der klinischen Forschung zur Phytotherapie relevant sind.

\section{Material und Methoden}

Einer der Mitbegründer der EbM, David Sackett, fasst 3 Kernaspekte im Prozess der evidenzbasierten Entscheidungsfindung zusammen: (EbM is) «the integration of (a) best re- search evidence with (b) clinical expertise and (c) patient values» [2]. Allerdings kam es, unter dem Druck eine Evidenzbasis zu schaffen, zur Fokussierung im Medizinsystem auf Punkt a), die beste Forschungsevidenz. Diese Einseitigkeit führte zur mangelhaften Berücksichtigung von Punkt b), klinischer Erfahrung, und Punkt c), Patientenwerten (z.B. Präferenzen, Bedenken, Erwartungen).

Aufgrund dieser Entwicklung der EbM werden drei provokante Hypothesen aufgestellt: 1. MAs in der Phytotherapie weisen methodische Schwächen bei den Auswahlkriterien der RCTs auf. 2. Oft sind Forschende an MAs beteiligt, die weder klinische Erfahrungen mit Phytotherapeutika noch ausreichende Kenntnisse zu phytotherapeutischen Arzneimitteln (Vielstoffgemischen) haben, noch Fachärzte für die untersuchte Grunderkrankung sind. 3. Der Nutzen der Ergebnisse phythotherapeutischer MAs wird nicht hinsichtlich Patientenwerten diskutiert.

Zur Überprüfung der Hypothesen wurden 3 Methoden angewandt: 1. Literaturrecherche zum Auffinden entsprechender Publikationen (Elektronische Datenbank: Pubmed, Suchbegriffe: Herbal medicine OR herbal drug OR phytotherapy OR herbal intervention OR herbal treatment, Limits: Meta-analysis, Ausschlusskriterium: Systematische Reviews, Einschlusskriterien: MAs mit RCTs/RTs zu pflanzlichen Arzneimitteln, Zeitraum: Beginn der Datenbank bis 2/2014), 2. Modifikation des QUORUM-Statements für MAs [20] entsprechend des CONSORT-Statements für RCTs für pflanzliche Interventionen [18]. 3. Diskussion aufgrund eigener Empirie mit MAs zu pflanzlichen Arzneimitteln.

\section{Ergebnisse}

\section{Datenrecherche}

Durch die Literatursuche wurden 359 Publikationen gefunden. Dabei fiel auf, dass nicht alle verfügbare Evidenz, d.h. publizierten MAs, auffindbar waren, auch wenn unter den Stichworten und im Abstrakt die Suchbegriffe auftauchten und die MAs über den Autorennamen in Pubmed auffindbar waren. [z.B. 21, 22]. Des Weiteren fanden sich unter den Publikationen systematische Reviews, die keine MAs waren, da das Datenpooling fehlte [z.B. 23-25]. Diese Zufallsbefunde werfen Fragen nach der Validität der Suchfilter bei Pubmed auf und zeigen eine Gefahr der Verfälschung der Ergebnisse durch eine Such- oder Filterverzerrung (search/filter bias). Daher müsste das NIH die Filtereinstellungen überprüfen.

\section{Forschungsevidenz}

Bezüglich der Forschungsevidenz zeigte sich, dass aufgrund der Auswahlverzerrung (selection bias) bei MAs die Heterogenität unter den eingeschlossen RCTs mitunter beachtlich ist. So wurden bei einer MA zu Johanniskrautextrakten RCTs mit einer Divergenz der Tagesdosierungen bis zum 7,5-Fachen und der Behandlungsdauer um das 3-Fache gepoolt. [26] Dieses 
Problem ist nicht nur bei phytotherapeutischen sondern auch bei konventionellen MAs zu finden (Divergenz in RCTs für MA zu Pentoxifyllin bei Behandlungsdauer bis zum 2-Fachen und der Dosierung bis zum 3-Fachen) [27]. Ob die Heterogenität auf bisher fehlende allgemeine Behandlungsstandards beim Durchführen der RCTs zurückgeht oder den metaanalytischen Ansatz, «soviele Daten wie möglich zu nutzen» [28], bleibt unklar. Aber soviel wie möglich an RCTs zur Reduktion des Publikationsbias einzubeziehen heißt auch, die mögliche Divergenz der Interventionsparameter in den einzelnen RCTs zu beachten, damit die Heterogenität nicht zu groß ist. Bei Johanniskrautextrakten jedoch wäre ein Versuch des Pooling der RCTs zusätzlich nach Subgruppen wie gegebenenfalls niedriger (200-500 mg), mittlerer (600-900 mg) und hoher Dosierung (1050-1800 mg) sowie Behandlungsdauer (4-8 oder 12 Wochen) aus klinischer Sicht sinnvoll.

Wo die Anzahl der publizierten RCTs klein ist und der Ansatz, soviel RCTs wie möglich in die MA einzubeziehen nicht möglich ist, haben manche Forschergruppen nicht die publizierten Daten, sondern die Rohdaten z.B. nach ITT reanalysiert, wenn die Interventionen (Behandlungsdauer, Tagesdosis, Messinstrument) nahezu identisch waren [z.B. 21, 22, 29, 30]. Dies zeigt große Unterschiede hinsichtlich der Homogenität in MAs.

\section{Klinische Erfahrung}

Bezüglich der in der EbM geforderten klinischen Erfahrung fiel bei manchen Publikationen auf, dass offenbar keine Fachärzte zwecks klinischer Erfahrung in Bezug auf die behandelte Grunderkrankung dabei waren [23-25, 31-33]. So stellt sich die Frage, ob die Schlussfolgerungen zur Forschungsevidenz im Rahmen klinischer Relevanz richtig interpretiert werden können. Des Weiteren stellt sich die Frage, ob die an der MA beteiligten Wissenschaftler detaillierte Kenntnisse mit pflanzlichen Arzneimitteln aufgrund einer entsprechenden Subspezialisierung oder eines Zertifikates haben (z.B. B.Sc. oder M.Sc. in Phytotherapie oder Fähigkeitsausweis Phytotherapie FMH oder Zertifikat Phytotherapie SMGP bzw. einer anderen Organisation) [23-26, 34], was auch bei den anderen MAs nicht aus der Publikation hervorging.

Da sich bereits zeigte, dass das CONSORT-Statement nicht ausreichend für RCTs mit Phytotherapeutika ist, wurde ein spezielles CONSORT für pflanzliche Interventionen verabschiedet [18]. Es scheint höchste Zeit, dies auch für MAs umzusetzen. Pflanzliche Arzneimittel weisen als Vielstoffgemische Besonderheiten auf, die ohne zusätzliche Ausbildung in Phytotherapie in der Regel nicht bekannt sind. Bei Johanniskrautextrakten [26] wäre, wegen des aus pharmakokinetischen Studien bekannten Interaktionspotentials, eine Subgruppenanalyse für hyperforinreiche und hyperforinarme Extrakte wichtig. Nur so könnte eine MA mit klinisch relevantem Datenpooling zu Wirksamkeit und Patientensicherheit in Abhängigkeit vom Hyperforingehalt entstehen.

\section{Patientenerwartung}

Bei allen genannten MAs waren keine Vertreter von Patientengruppen beteiligt, die Aspekte zu Patientenerwartungen (Präferenz, Bedenken, Werte) hätten mit einbringen können [21, 22, 26-32]. Bei nur einer MA wurde überhaupt minimaler Bezug zu möglichen Patientenerwartungen genommen [28].

\section{Diskussion}

Die schnelle Übernahme der Methodik der EbM durch Forschende in der Komplementärmedizin hat vor allem für phytotherapeutische Interventionen in den letzten 20 Jahren eine Fülle von MAs ermöglicht. Hierdurch konnte es verhindert werden, dass die Phytotherapie in der klinischen Forschung den Anschluss an den methodischen Mainstream verlor, weil eine Fülle von Forschungsevidenz zur Verfügung steht. Doch nun ist es an der Zeit, diese Forschungsevidenz selbstkritisch zu analysieren.

Es scheint unlogisch, über die Integration von Komplementärmedizin im Medizinsystem zu sprechen und so zu tun, als sei dies bei ausreichender Evidenz mit der Übernahme einzelner Therapien in Behandlungsrichtlinien getan. Kein einzelner Arzt und kein einzelner Wissenschaftler kann bei der heutigen Datenmenge alleine integrativ arbeiten. Deshalb ist auch bei der klinischen Forschung, beim Erstellen von RCTs und MAs die inter- und transdisziplinäre Zusammenarbeit klinischer Pharmakologen oder sonstiger Spezialisten für MAs mit Fachärzten, zertifizierten Komplementärmedizinern (z.B. Phytotherapeuten) und auch mit Patientenvertretern notwendig. Dass MAs und SRs hauptsächlich von nichtärztlichen Wissenschaftlern erstellt werden, die teils keine Erfahrungen in der Behandlung der Patienten mit der untersuchten Intervention haben, reduziert die Möglichkeit, klinisch relevante Aussagen treffen zu können. Für EbM und Komplementärmedizin wird daher von unterschiedlicher Seite zu Recht klinische Erfahrung gefordert $[1,2,5]$.

Darüber hinaus muss das QUORUM-Statement für MAs für pflanzliche Interventionen weiterentwickelt werden:1. Aufgrund der dargestellten Daten müssen in MAs die pflanzlichen Interventionen genau definiert werden, um zu vermeiden, dass divergierende pflanzliche Vielstoffgemische mit unterschiedlichem Wirkungs- und Nebenwirkungspotential miteinander gepoolt werden. Daher sind Phytotherapieexperten an phytotherapeutischen Metaanalysen zu beteiligen. 2. Trotz geringer Studienlage sollen nicht RCTs mit deutlich divergierenden und daher klinisch relevanten Tagesdosierungen miteinander gepoolt werden. Hierdurch sind Funnel Plots mit geringer Studienzahl zu tolerieren. 3. Aus dem gleichen Grund sollen RCTs mit deutlich divergierender Behandlungsdauer nicht gepoolt werden. 4. Fachärzte müssen an der Erstellung von Metaanalysen beteiligt sein, um die klinisch relevante Darstellung zu gewähren. 


\section{Literatur}

1 Sackett DL, Rosenberg WM, Gray JA, Haynes RB, Richardson WS: Evidence based medicine: what it is and what it isn't. BMJ 1996;312:1-72.

2 Sackett DL, Straus SE, Richardson WS, Rosenberg W, Haynes RB: Evidence-Based Medicine. How to practice and teach EBM, ed 2. Edinburg, Churchill Livingstone, 2000.

3 Feinstein AR: Evidence-based medicine; in Warrell DA, Cox TM, Firth JD, Benz Jr EJ (eds): Oxford Textbook of Medicine, ed 4. New York, Oxford University Press, 2003, pp 21-24.

4 Kunz R, Ollenschläger G, Raspe H, Jonitz G, Kolkmann FW: Lehrbuch Evidenzbasierte Medizin in Klinik und Praxis. Köln, Deutscher Ärzte-Verlag, 2000.

5 Ernst E: Complementary medicien: too good to be true? J R Soc Med 1999;92:1-2.

6 Greenhalgh T: Einführung in die evidence-based medicine: kritische Beurteilung klinischer Studien als Basis einer rationalen Medizin, ed 2. Bern, Huber, 2003.

7 Sterne JAC, Egger M, Smith GD: Investigating and dealing with publication and other biases in meta-analysis. BMJ 2001;323:1001-1005.

8 Morling JR, Maxwell H, Stewart M: Padma 28 for intermittent claudication. Cochrane Database Syst Rev 2013;7:CD007371.

9 Sweeting MJ, Sutton AJ, Lambert PC: What to add to nothing? Use and avoidance of continuity corrections in meta-analysis of sparse data. Stat Med 2004;23: 1351-75.

10 Berlin JA, Laird NM, Sacks HS, Chalmers TC: A comparison of statistical methods for combining event rates from clinical trials. Stat Med 1989;8:141-51.

11 Biller-Andorno N: Evidenzbasierte Gesundheitsversorgung - kritische Nachfragen vor ethischem Hintergrund. Wien Med Wochenschr 2002;152:161.

12 Holmes D, Murray SJ, Perron A, Rail G: Deconstructing the evidence-based discourse in health sciences: truth, power and fascism. Int J Evid Based Healthc 2006;4:180-6.

13 James N: Evidence based medicine. Scientific method and raw data should be considered. BMJ 1996;313:169-170.

14 Greenhalgh T, Russell J: Evidence-based policymaking: a critique. Perspect Biol Med 2009;52:304-18.

15 Smith ML, Glass GV: Meta-analysis of psychotherapy outcome studies. Am Psychol 1977;32:752-60

16 Wong G, Greenhalgh T, Westhorp G, Buckingham J, Pawson R: RAMESES publication standards: meta-narrative reviews. BMC Medicine 2013;11:20.

17 Moher D, Schulz KF, Altman DG, et al: The CONSORT statement: revised recommendations for improving the quality of reports of parallel group randomized trials. Ann Intern Med 2001;17:657-662.

18 Gagnier JJ, Boon H, Rochon P, Moher D, Barnes J, Bombardier C; CONSORT Group: Recommendations for reporting randomized controlled trials of herbal interventions: Explanation and elaboration. J Clin Epidemiol 2006;59:1134-49.

19 Gagnier JJ, Moher D, Boon H, Beyene J, Bombardier C: Randomized controlled trials of herbal interventions underreport important details of the intervention. J Clin Epidemiol 2011;64:760-9.

20 Moher D, Cook DJ, Eastwood S, Olkin I, Rennie D, Stroup DT, for the QUOROM Group: Improving the quality of reports of meta-analyses of randomised controlled trials: the QUOROM statement. Quality of reporting of meta-analyses. Lancet 1999;354:1896-900

21 Melzer J, Brignoli R, Diehm C, Reichling J, Do D-D, Saller R: Treating intermit tent claudication with Tibetan medicine Padma 28: does it work? Atherosclerosis 2006;189:39-46.

22 Melzer J, Saller R, Schapowal A, Brignoli R: Systematic review of clinical data with BNO-101 (Sinupret) in the treatment of sinusitis. Forsch Komplementmed 2006;13:78-87.

23 Stevinson C, Huntley A, Ernst E: A systematic review of the safety of kava extract in the treatment of anxiety. Drug Saf 2002;25:251-61.

24 Perry R, Terry R, Watson LK, Ernst E: Is lavender an anxiolytic drug? A systematic review of randomised clinical trials. Phytomedicine 2012;19:825-35.

25 Stevinson C, Ernst E: Valeria for insomnia: a systematic review of randomized clinical trials. Sleep Med 2000;1:91-99.

26 Linde K, Berner MM, Kriston L: St John's wort for major depression. Cochrane Database Syst Rev 2008:CD000448.

27 Girolami B, Bernardi E, Prins MH, Ten Cate JW, Hettiarachchi R, Prandoni P, Girolami A, Buller HR: Treatment of intermittent claudication with physical training, smoking cessation, pentoxifylline, or nafronyl: a meta-analysis. Arch Intern Med 1999:159:337-45.

28 Robertson C, Idris NR, Boyle P: Beyond classical meta-analysis: can inadequately reported studies be included? Drug Discov Today 2004;9:924-31.

29 Melzer J, Rösch W, Reichling J, Brignoli R, Saller R: Meta-analysis: phytotherapy of functional dyspepsia with STW 5 (Iberogast ${ }^{\circledR}$ ). Aliment Pharm Ther 2004;20 1279-87.
30 Uehleke B, Brignoli R, Rostock M, Saller R, Melzer J: Phytodolor ${ }^{\circledR}$ in musculo-skeletal disorders: re-analysis and meta-analysis. Forsch Komplementmed 2011;18:249-56.

31 Pittler MH, Ernst E: Kava extract for treating anxiety. Cochrane Database Syst Rev 2003:CD003383.

32 Schulz V: New therapeutic studies and meta-analysis. St. John>s wort extract vs. synthetics. Pharm Unserer Zeit 2003;32:228-34

33 Agbabiaka TB, Guo R, Ernst E: Pelargonium sidoides for acute bronchitis: a systematic review and meta-analysis. Phytomedicine 2008;15:378-85.

34 Hausenblas HA, Saha D, Dubyak PJ, Anton SD: Saffron (Crocus sativus L.) and major depressive disorder: a meta-analysis of randomized clinical trials. J Integr Med 2013;11:377-83.

\section{Versorgungsforschung - ein wichtiges Instrument der Zukunft zum Beleg des Nutzens von (pflanzlichen) Arzneimitteln}

\author{
Autor: Günter Meng
}

\section{Hintergrund}

Ein Patient erwartet von seinem Arzt oder Apotheker, dass die Therapie, die diese ihm empfehlen, ihm hilft. Und zwar genau ihm, in seinem Alltag, und zwar so, dass er oder sie eine relevante Erleichterung der wahrgenommenen Beschwerden erfährt.

Dabei ist es diesem Patienten erst einmal gleichgültig, ob es in Studien Gruppen von Patienten gegeben hat, bei denen die Therapie im Vergleich mit einer Alternative im Mittel einen Erfolg gezeigt hat. Allerdings erwartet er natürlich, dass die ausgesprochene Empfehlung auf der Basis neuester wissenschaftlicher Erkenntnisse gegeben wird, dass der Empfehlende auf dem Hintergrund seiner eigenen beruflichen Erfahrung mit ganzer Überzeugung hinter dieser Empfehlung steht und dass auch seine eigenen, ganz persönlichen Präferenzen angemessen berücksichtigt werden.

Diese Erwartung ist ziemlich genau das, was David Sackett [1] mit dem Begriff «Evidenzbasierte Medizin (EBM)» belegt hat: «EBM ist der gewissenhafte, ausdrückliche und vernünftige Gebrauch der gegenwärtig besten externen, wissenschaftlichen Evidenz für Entscheidungen in der medizinischen Versorgung individueller Patienten. Die Praxis der EBM bedeutet die Integration individueller klinischer Expertise mit der bestverfügbaren externen Evidenz aus systematischer Forschung.»

Und auch diese Definition spiegelt wohl nur wider, was einfach eine kunstgerechte, ethisch verantwortete Ausübung der Heilkunst ist, wie sie beispielsweise in dem medizinethischen Vier-Prinzipien-Modell von Beauchamp und Childress [2] Ausdruck findet:

- Respekt vor der Autonomie des Patienten

- Nicht-Schaden

- $\quad$ Fürsorge, Hilfeleistung

- Gleichheit und Gerechtigkeit 
Es geht hier also um eine erfahrbare therapeutische Wirkung unter den Bedingungen des Alltags medizinischer Versorgung. Und diese Bedingungen unterscheiden sich bekanntlich ganz erheblich von denjenigen, die in randomisierten Doppelblindprüfungen (RCT) gewählt werden.

\section{Praxisforschung mit RCT?}

RCTs jedoch sind der Goldstandard der therapeutischen Wirksamkeitsforschung. Nur in RCTs lässt sich «spezifische therapeutische Wirksamkeit» kausal belegen. Und auch wenn das Modell der spezifischen therapeutischen Wirksamkeit für den Patienten erst einmal von untergeordneter Bedeutung ist, denn ihn interessiert die Gesamtwirkung, so ist es für die $\mathrm{Zu}-$ lassung von Arzneimitteln zum Markt und auch für die zurzeit angewandten Modelle der formellen Nutzenbewertung von entscheidender Bedeutung.

Dies hat gute Gründe, die hier nicht erörtert werden können (siehe hierzu aber z.B. [3]). Aber auch wenn diese randomisierten Studien so praxisnah wie nur möglich konzipiert sind, so haben sie doch immer eine ganz entscheidende Abweichung vom therapeutischen Alltag. Denn dieser sollte ja von den oben genannten Prinzipien geprägt, also so gestaltet sein, dass der Nutzen, den der Patient aus der gesamten Therapiesituation zieht, maximal ist. Und dazu gehört, wie gut belegt ist [3], ein gutes Vertrauensverhältnis zwischen Arzt und Patient. Ein RCT jedoch ist sozusagen der institutionalisierte «Bruch dieses Vertrauens». Und dies wird insbesondere durch die freilich absolut notwendige ausführliche und detaillierte Aufklärung des Patienten auch über die Randomisierung verstärkt [4].

Aus diesen Überlegungen wird deutlich, dass die Untersuchung der Wirksamkeit eines Arzneimittels durch RCTs zwar eine notwendige Voraussetzung für ihre Zulassung zum Markt ist, dies nach allgemeiner wissenschaftlicher Überzeugung unserer Zeit, sie lässt jedoch nur sehr beschränkte Aussagen darüber zu, welchen Nutzen ein Patient in einer guten therapeutischen Alltagsversorgungssituation aus dieser Therapie erwarten kann.

Dies ist das Feld der Versorgungsforschung.

\section{Versorgungsforschung}

Der Begriff «Versorgungsforschung» wird z.B. in Deutschland sehr häufig im Zusammenhang mit Untersuchungen zur Nutzenbewertung bei der Preisfestsetzung im öffentlichen Gesundheitssystem gebraucht. Dies ist allerdings nur eine, wenn auch wichtige, Fragestellung für Forschungsmethoden, die sich mit der realen Versorgungswirklichkeit befassen. Dass der Begriff sehr viel breiter zu verstehen ist, zeigt beispielsweise eine von der Deutschen Bundesärztekammer veröffentlichte Monographie [5]: «Versorgungsforschung ist die wissen- schaftliche Untersuchung der Versorgung von Einzelnen und der Bevölkerung mit gesundheitsrelevanten Produkten und Dienstleistungen unter Alltagsbedingungen».

Ist ein Arzneimittel also erst einmal den gesetzlichen Anforderungen entsprechend, in der Regel auf der Basis von positiven Ergebnissen aus RCTs zum Markt zugelassen, dann sind es die Methoden der Versorgungsforschung, die sein Potenzial für den Patienten erst praxisnah erfassen können. Und dieses Potenzial umfasst sowohl die erwünschten wie die nicht erwünschten Wirkungen als auch die Frage, welche Patienten mehr als andere profitieren können und wie eine konkrete Therapie sich in das Umfeld der insgesamt zur Verfügung stehenden therapeutischen Alternativen möglichst gut zum Nutzen des Patienten einpassen lässt. Und auch dies sind nur Überschriften.

Es liegt auf der Hand, dass sich zur Untersuchung dieser Fragestellungen besonders solche Studienmethoden eignen, die nicht oder möglichst wenig in den Versorgungsalltag intervenieren, nichtinterventionelle Studien, auch Beobachtungsstudien, observational studies genannt [6].

Die EU-Arzneimittelgesetzgebung trägt dem seit einigen Jahren Rechnung, indem sie das Instrument der nichtinterventionellen Studie definiert [7]. Und in diesem Zusammenhang wurden entsprechend auch detaillierte fachliche Empfehlungen z.B. für die Gestaltung des Studienplans und die Wahl der Auswertungsverfahren derartiger epidemiologischer Studien veröffentlicht [8].

Für Phytopharmaka ist dies eine wichtige Entwicklung. Denn für Produkte - seien sie voll zugelassen oder auf Basis ihrer langjährigen Anwendung registriert -, die schon längere Zeit im Markt sind, ist es essenziell, Erkenntnisse bei deren Anwendung unter alltäglichen Bedingungen zu sammeln und dabei auch zu erfassen, wie sich diese Therapieverfahren jeweils neu vor dem sich immer wandelnden Hintergrund der aktuellen medizinischen Methoden einordnen lassen. Es bedarf keiner besonderen Erwähnung, dass hierbei Studien auf höchstem wissenschaftlichem und technischem Niveau mit umfangreicher Qualitätssicherung der Durchführung und optimalen Vorkehrungen für die Kontrolle oder Vermeidung systematischer Verzerrungen (Bias) gemeint sind, die insbesondere den oben genannten Anforderungen entsprechen.

\section{PhytoVIS}

Ein Beispiel für ein solches Projekt ist die Datenbank PhytoVIS der deutschen Kooperation Phytopharmaka [9]. Hierbei wurde eine Plattform erarbeitet, mit deren Hilfe einerseits eine grundlegende wissenschaftliche Langzeiterfassung von Phytopharmaka-Nutzungsdaten hinsichtlich erlebter Wirkung und erlebter relevanter Nebenwirkungen möglich ist (Studienleitung: Prof. Mösges, Universität Köln, Publikation zur Pilotphase in Vorbereitung) und andererseits speziellere Versorgungsforschungsprojekte insbesondere aus akademischen Forschung erleichtert werden. 
Das Projekt PhytoVIS ist in den vergangenen Monaten aus der Konzeptions- und Pilotphase in die Routineanwendung eingetreten. Es steht also nun der Nutzung durch Wissenschaftler offen, sofern das entsprechende Projekt von einer unabhängigen Prüfungskommission, die durch die Kooperation beauftragt wurde, befürwortet wird. Daneben sehen die von der Kooperation Phytopharmaka festgelegten Nutzungsstatuten für das Werkzeug PhytoVIS [9] vor, dass strikte Qualitätssicherungs- und Transparenzbedingungen eingehalten werden müssen. Einzelheiten zur Gestaltung dieses Projekts werden im Vortrag ausführlicher dargelegt.

PhytoVIS ist also ein Werkzeug, das Studienprojekte mit Fragestellungen zur Versorgungsforschung hinsichtlich der praktischen Anwendung pflanzlicher Arzneimittel fördert und erleichtert. Solche Projekte sind besonders wichtig, um vor dem sich schnell ändernden Hintergrund der aktuellen medizinischen Möglichkeiten pflanzliche Arzneimittel entsprechend den Prinzipien einer evidenzbasierten Medizin im eingangs dargelegten Sinne zum Nutzen des Patienten gebrauchen zu können.

\section{Literatur}

1 Sackett DL, Rosenberg WMC, Gray JAM, Haynes RB, Richardson WS: Was is Evidenzbasierte Medizin und was nicht? Münch med Wschr 1997;139:644-645.

2 Beauchamp TL, Childress JF: Principles of Biomedical Ethics, ed 7. Oxford, Oxford University Press, 2012.

3 Jakobsen JC, Gluud C: The Necessity of Randomized Clinical Trials. Br J Med Med Res 2013;3:1453-1468

4 Gaus W, Muche R: Is a controlled randomised trial the non-plus-ultra design? A contribution to discussion on comparative, controlled, non-randomised trials. Contemp Clin Trials 2013;35:127-132.

5 Arbeitskreis «Versorgungsforschung» beim wissenschaftlichen Beirat: Rahmenkonzept zur Förderung der Versorgungsforschung durch die Bundesärztekammer. Bundesärztekammer 2004.

6 Röhrig B, du Prel JB, Wachtlin D, Blettner M: Studientypen in der medizinischen Forschung. Dtsch Arztebl Int 2009;106:262-8.

7 Richtlinie 2001/20/Eg des europäischen Parlaments und des Rates vom 4. April 2001 zur Angleichung der Rechts- und Verwaltungsvorschriften der Mitgliedstaaten über die Anwendung der guten klinischen Praxis bei der Durchführung von klinischen Prüfungen mit Humanarzneimitteln. www.eur-lex.europa.eu.

8 The European Network of Centres for Pharmacoepidemiology and Pharmacovigilance (ENCePP). Guide on Methodological Standards in Pharmacoepidemiology (Revision 2).EMA/95098/2010.www.encepp.eu/standards_and_guidances

9 Wegener T: PhytoVIS ${ }^{\circledR}$ - eine Versorgungsforschungs-Datenbank der Kooperation Phytopharmaka - Statuten. 2013.www.phytovis.de.

\section{Bryophyllum pinnatum: Klinische Anwendung bei hyperaktiven Zuständen unterschiedlicher Genese}

\section{Autoren: Ursula von Mandach, Karin Fürer**}

Bryophyllum pinnatum (Lam.) Oken (Synonym: Kalanchoe pinnata (Pers.), eine krautartige Pflanze mit glatten, fleischigen, unbehaarten Blättern, gehört zur Familie der Crassulaceae (Abb. 1). Das natürliche Hauptvorkommen von Bryophyllum liegt in Madagaskar. Je nach Auszug (Presssaft bzw. wässriger oder alkoholischer Extrakt), Provenienz und Pflanzenteil variieren die Inhaltsstoffe. Eine Charakterisierung des Blattpresssaftes, des wässrigen sowie methanolischen Extraktes von B. pinnatum durch unsere Studiengruppe hat gezeigt, dass Flavonoide zu den Hauptinhaltsstoffen gehören und nur geringe Mengen an herzwirksamen Bufadienoliden vorkommen [1,2]. Kürzlich wurden 4 Bufadienolide im ethanolischen Extrakt von B. pinnatum (Herkunft: Deutschland und Brasilien) quantifiziert: gesamthaft $5,31 \mathrm{mg} / 100 \mathrm{~g}$ Trockengewicht, respektive 14,28 mg/100 g (zum Vergleich: 41,11 $\mathrm{mg} / 100 \mathrm{~g}$ in der Species B. daigremontianum) [3].

Bryophyllum wurde von Rudolf Steiner, dem Begründer der Anthroposophischen Medizin, bereits 1921 für die Behandlung von damals noch «Hysterie» genannten Unruhezuständen empfohlen. Aus der Bezeichung Hysterie lassen sich verschiedene Unruhe- bzw. hyperaktive Zustände als mögliche Indikationen für B. pinnatum ableiten. Dazu gehören z.B. Einschlafstörungen, unter anderem im Rahmen des Aufmerk-

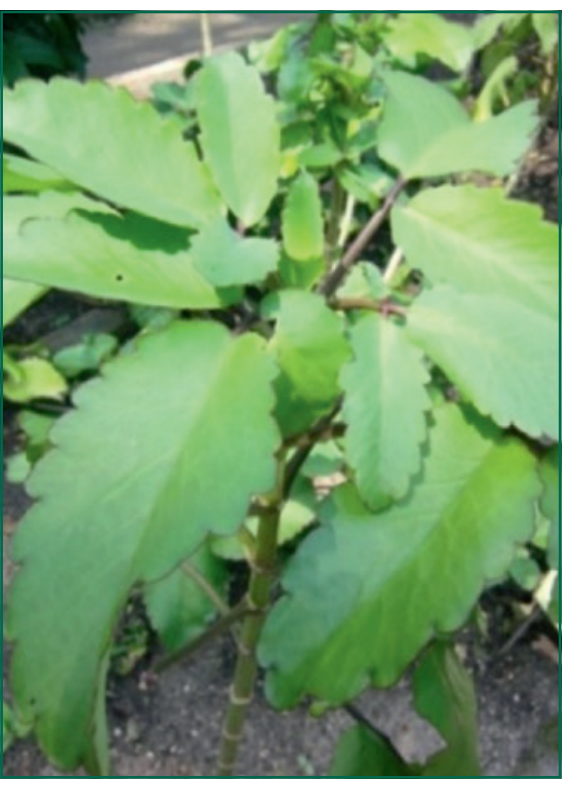

lum pinnatum.

* In Vertretung der Bryophyllum Study Group: Betschart C, Brenneisen R, Fürer K, Hamburger M, Mennet-von Eiff M, Potterat O, Schnelle M, Simões-Wüst AP, von Mandach U. 
samkeitsdefizit-/Hyperaktivitätssyndroms, ADHS, sowie speziell in der Geburtshilfe und Gynäkologie die vorzeitige Wehentätigkeit und die hyperaktive Blase.

In einer jüngsten Anwendungsbeobachtung unserer Studiengruppe verbesserte $B$. pinnatum bei 42 Schwangeren die Schlafqualität und verminderte die Anzahl von Aufwachphasen [4]. Die Applikation von Bryophyllum erfolgte oral, die tägliche Dosis (3-6 Tabletten zu $350 \mathrm{mg}$ ) liegt tiefer als bei der Tokolyse (4-8 Tabletten/Tag). Zur Behandlung der vorzeitigen Wehentätigkeit wird B. pinnatum seit 1970 in den anthroposophischen Geburtskliniken mit großem Erfolg eingesetzt, sowohl intravenös als auch oral [5, 6]. Bisher wurden in der Schweiz im Rahmen eines prospektiven, randomisierten, klinischen Studiendesigns nach Vorgaben des Schweizerischen Heilmittelgesetzes 2 Studien durchgeführt: 1. eine doppelblinde Studie mit B. pinnatum oral vs. Placebo zusätzlich bei Patientinnen mit einer prophylaktischen Tokolyse (synthetisches Tokolytikum) und 2. eine multizentrische Studie zum Vergleich von B. pinnatum versus das Off-Label-Tokolytikum Nifedipin, bei der Akutpatientin mit portiowirksamen Wehen; eine Auswertung bei 27 Patientinnen zeigte eine mit Nifedipin vergleichbare Wirksamkeit von Bryophyllum.

In mehreren Versuchsanordnungen von humanen Myometriumproben in einem Organbad konnten wir zeigen, dass $B$. pinnatum (als wässriger Blattextrakt bzw. Blattpresssaft) spontane als auch Oxytocin-induzierte Kontraktionen hemmt, dies vor allem in ihrer Stärke (Amplitude) [1,7]. Darüber hinaus konnten wir nachweisen, dass $B$. pinnatum den Oxytocin-induzierten Anstieg von Calcium in humanen Myometriumzellen dosisabhängig hemmt [8], was darauf hindeutet, dass Bryophyllum ganz spezifisch an den regulatorischen Prozessen der myometrialen Kontraktilität angreift.

Die sedierende Wirkung sowie die an der Uterusmuskulatur gezeigte relaxierende Wirkung von $B$. pinnatum prädestinieren B. pinnatum als phytotherapeutischen Ansatz bei der Behandlung der hyperaktiven Blase. Eine erste klinische Studie mit dieser Fragestellung zeigte tatsächlich einen Effekt zugunsten von B. pinnatum (Reduktion der Anzahl Miktionen pro $24 \mathrm{~h}$ bei B. pinnatum in 8 von 10 Patienten $(\mathrm{p}=0,037)$ bzw. in der Placebogruppe bei 5 von 9 Patienten $(p=0,89)$ ) [9]. Eine Nachfolgestudie ist bereits von Swissmedic notifiziert.

In einer In-vitro-Versuchsanordnung mit Schweineblasen (Detrusormuskelstreifen) vermochte B. pinnatum-Blattpresssaft mit der höchsten getesteten Konzentration (10\%) den durch Carbachol kontrahierten Muskel um 20\% zu relaxieren ( $\mathrm{p}<0,05)$. Zudem hemmte der Presssaft $(5 \%)$ die Auslösung der Muskelkontraktion mittels Elektrofeldstimulation um knapp 70\% [10]. Laufende Experimente mit einer aus dem methanolischen Extrakt isolierten Flavonoidfraktion (0,1-1 $\mathrm{mg} / \mathrm{ml}$ ) zeigen eine zeit- und dosisabhängige Hemmung der ursprünglichen Kontraktilität um bis zu $80 \%$. Weiterführende Untersuchungen sollen die Wirkmechanismen bzw. Rezeptoren in der Blase identifizieren, die für diese Wirkungen verantwortlich sind.

\section{Literatur}

1 Wächter R, Brenneisen R, Hamburger M, Mennet M, Schnelle M, Worel AM, Simões-Wüst AP, von Mandach U: Leaf press juice from Bryophyllum pinnatum (Lamarck) Oken induces myometrial relaxation. Phytomedicine 2011;19:74-82.

2 Fürer K, Raith M, Brenneisen R, Mennet M, Simões-Wüst AP, von Mandach U, Hamburger M, Potterat O: Two new flavonol glycosides and a metabolite profile of Bryophyllum pinnatum, a phytotherapeutic used in obstetrics and gynaecology. Planta Med 2013;79:1565-1571.

3 Gerodetti M: Quantification of bufadienolides in extracts and preparations of Bryophyllum pinnatum. Master thesis University of Basel, 2013.

4 Lambrigger-Steiner C, Simões-Wüst AP, Kuck A, Fürer K, Hamburger M, von Mandach U: Sleep quality in pregnancy during treatment with Bryophyllum pinnatum: An observational study. Phytomedicine 2013;in press.

5 Vilaghy I: Decreasing the rate of premature delivery with phytotherapy - results from general practice. Ther Umsch 2002;59:696-701.

6 Plangger N, Rist L, Zimmermann R, von Mandach U: Intravenous tocolysis with Bryophyllum pinnatum is better tolerated than beta-agonist application. Eur J Obstet Gynecol Reprod Biol 2006;124:168-172.

7 Gwehenberger B, Rist L, Huch R, von Mandach U: Effect of Bryophyllum pinnatum versus fenoterol on uterine contractility. Eur J Obstet Gynecol Reprod Biol 2004:113:164-171.

8 Simões-Wüst AP, Grãos M, Duarte CB, Brenneisen R, Hamburger M, Mennet M, Ramos MH, Schnelle M, Wächter R, Worel AM, von Mandach U: Juice of Bryophyllum pinnatum (Lam.) inhibits oxytocin-induced increase of the intracellular calcium concentration in human myometrial cells. Phytomedicine 2010;17:980986.

9 Betschart C, von Mandach U, Seifert B, Scheiner D, Perucchini D, Fink D, Geissbühler V: Randomized, double-blind placebo-controlled trial with Bryophyllum pinnatum versus placebo for the treatment of overactive bladder in postmenopausal women. Phytomedicine 2013;20:351-358.

10 Schuler V, Suter K, Fürer K, Eberli D, Horst M, Betschart C, Brenneisen R, Hamburger M, Mennet M, Schnelle M, Simões-Wüst AP, von Mandach U: Bryophyllum pinnatum inhibits detrusor contractility in porcine bladder strips - a pharmacological study towards a new treatment option of overactive bladder. Phytomedicine 2012;19:947-951.

\section{Pleiotrope Signaturen - eine Anregung zur Aufwertung traditioneller pflanzlicher Arzneimittel}

\section{Autoren: Herbert Schwabl, Cécile Vennos, Reinhard Saller}

\section{Phytotherapie als empirische Wissenschaft}

Schon seit Beginn der Menschheit waren Pflanzen nicht nur Teil der Ernährung, sondern wurden auch für medizinische Zwecke verwendet. Historische Quellen geben über die Jahrhunderte hinweg eine Vielzahl von Berichten und praktischen Anweisungen zum medizinischen Einsatz von Heilkräutern.

Dieses Wissen wurde oft in einer heute anekdotisch erscheinenden Form aneinandergereiht, wobei den Pflanzen und pflanzlichen Rezepturen schon von Beginn an eine Vielzahl von Indikationen und Wirkmechanismen zugeschrieben wurde. Später entwickelten sich insbesondere in Europa und Asien verschiedene traditionelle Medizinsysteme mit ihrer zum Teil hoch entwickelten Ätiologie und Methodik der Diagnose und Therapie. Letztere basierte hauptsächlich auf einer hoch differenzierten, auf Heilpflanzen basierenden Materia Medica. 
Mit dem Aufkommen der Naturwissenschaften geriet diese breit gefächerte Wissensbasis zunehmend ins Abseits. Die moderne Forschung fokussiert auf kausale Intervention und auf klare Beschwerdebilder. In der Phytotherapie zeigte sich aber schon bald die Begrenztheit des Ansatzes, der auf einige wenige spezifische Liganden und kausale Mechanismen setzt, denn die Wirkung einer Heilpflanzenzubereitung lässt sich nur in Ausnahmefällen anhand spezifischer Moleküle klassieren. Immerhin gelang es, für eine Reihe von Indikationen pflanzlicher Arzneimittel die Evidenzpyramide zu schliessen. («Pflanzliche Arzneimittel» und «Phytoarzneimittel» wird in diesem Artikel synonym verwendet für Einzeldrogen bzw. Einzelextrakte wie auch für Drogen- und Extraktkombinationen.)

Heute wie damals werden aber Phytoarzneimittel hauptsächlich in der Selbstmedikation sowie in der Allgemeinpraxis eingesetzt. Pflanzliche Arzneimittel kommen aufgrund der guten Verträglichkeit und des im Allgemeinen geringen Nebenwirkungspotentials vor allem bei Kindern zum Einsatz, bei Befindlichkeitsstörungen und lebensbegleitend bei chronischen Erkrankungen. Ihre besondere Stärke finden Phytoarzneimittel bei der Behandlung von multimorbiden Zuständen, bei komplexen Krankheitsbildern bzw. Therapiesituationen. Das gesichtete Erfahrungswissen im Sinne einer wissenschaftlich tragfähigen Empirie beschreibt die Wirkung von pflanzlichen Arzneimitteln als systemisch unter Einbezug großer Funktionskreise, angepasst an komplexe Therapiesituationen, oft aufgewertet durch einzelne Fallberichte.

Dagegen wird die klinische Forschung mit pflanzlichen Arzneimitteln immer aufwändiger, sei es durch die Anforderungen der Good Clinical Practice, die Ethikrichtlinien oder die Empfehlungen der spezialisierten Fachgesellschaften. Die immer komplizierteren Studiendesigns und die damit einhergehende Verteuerung und nicht angemessene, oft sogar unsinnige Erschwernis der klinischen Studien führt dazu, dass immer weniger groß angelegte Studien durchgeführt werden können. Eine Zulassung des gesamten therapeutischen Potentials einer Arzneispezialität z.B. als gut etabliertes Arzneimittel ist daher, wenn überhaupt, nur für wenige Arzneipflanzenzubereitungen möglich.

Die große Mehrheit der traditionell eingesetzten pflanzlichen Wirkstoffe und Pflanzengemische kann diese Anforderungen auf Grund mangelnder ökonomischer Ressourcen nicht erfüllen. Dagegen steht das unausgeschöpfte Potential eines auch heute noch wachsenden Körpers an wissenschaftlich tragfähigen empirischen Daten zur Anwendung pflanzlicher Arzneimittel.

\section{Traditionelle pflanzliche Arzneimittel}

In der Europäischen Union ermöglichte erst die Richtlinie 2004/24/EC, die vor genau 10 Jahren in Kraft gesetzt wurde, das empirische Wissen um die Verwendung von Pflanzen in das moderne Arzneimittelrecht zu integrieren. Diese Richtli- nie führte die Kategorie der traditionellen pflanzlichen Arzneimittel ein, die es erlaubt an die empirische Überlieferung anzuschließen. Damit ist es im Prinzip möglich, das gewachsene empirische Wissen über die Anwendung pflanzlicher Zubereitungen in das moderne Arzneimittelrepertoire überzuführen und damit aufzuwerten. Das Europäische Committee on Herbal Medicinal Products (HMPC) hat inzwischen die Herkulesaufgabe bewältigt, für mehr als 100 Arzneipflanzen verbindliche Monografien zu verfassen. Ferner werden wegweisende Richtlinien zur Qualität pflanzlicher Arzneimittel publiziert.

So wichtig die Errungenschaft der Richtlinie 2004/24/EC auch ist, sie hat allerdings auch einige Schwächen, die nur vom Gesetzgeber zu lösen sind.

- Die traditionelle Anwendung ist für insgesamt 30 Jahre zu belegen, davon 15 Jahre innerhalb der Europäischen Gemeinschaft. Dies schließt außereuropäische Medizintraditionen aus (und nota bene die Schweiz). Generell müssen komplementärmedizinische Therapiesysteme mehr berücksichtigt werden.

- Das Spektrum der möglichen Indikationen muss erweitert werden über die zur Zeit möglichen einfachsten Erkrankungen hinaus. Auch die Anforderungen an die Sicherheit müssen praxisgerecht werden, um das im Allgemeinen positive Nutzen-/Risikoverhältnis bei traditionellen Arzneimitteln besser abzubilden.

- Patent-/Datenschutz ist ungelöst, daher fehlt auch der Anreiz, kostspielige Forschung zu betreiben.

- Die Grenze zwischen Nahrungsergänzungen und Arzneimitteln ist unscharf. Nahrungsergänzungen können nahezu ohne Registrierungsaufwand auf den Markt gebracht werden, oft mit zum Teil sehr attraktiven Auslobungen. Arzneimittel haben zwar einerseits eine höhere Wirkstärke mit gesicherter Indikation, haben aber gleichzeitig den Nachteil, dass die zugebilligte Indikation eher wenig attraktiv erscheint und in der Arzneimittelinformation mitunter mehr Warnhinweise zu lesen sind als positive Anwendungsempfehlungen. $\mathrm{Ob}$ im Zeitalter der transnationalen Internet-Information für einen durchschnittlichen Verbraucher der Unterschied der verschiedenen Produktkategorien ins Bewusstsein dringt, ist stark anzuzweifeln. Hier ist zu befürchten, dass eine Reihe bewährter Arzneipflanzen in die Kategorie der Nahrungsergänzungen abwandert.

Dagegen wächst das Bedürfnis nach komplementärmedizinischen Methoden, insbesondere nach pflanzlichen Arzneimitteln. Dies umso mehr, als mit dem zunehmenden Älterwerden der Bevölkerung in den Industriestaaten die Inzidenz für Multimorbidität, Komorbidität und Alterserkrankungen steigt.

Für die an einer qualitativ hochwertigen Phytotherapie interessierten Kreise stellt sich also die Frage, wie pflanzliche Arzneimittel und insbesondere die Kategorie der traditionellen Arzneimittel im Rahmen der heute bestehenden Vorschriften 
aufgewertet werden können. Ermöglichen könnte dies eine erweiterte Beurteilung der Plausibilität der Wirksamkeit oder die Erweiterung der möglichen Anwendungsgebiete.

\section{Multi-Target-Drugs in der therapeutischen Anwendung}

Herkömmlich wird die Wirkstoffcharakteristik eines pflanzlichen Vielstoffgemisches unter dem Aspekt der Einzeldrogen bzw. Einzelextrakte analysiert. Chemisch gesehen sind Phytotherapeutika Vielstoffgemische, strukturell liegen die einzelnen Wirkstoffkomponenten in meist sehr niedrigen Konzentrationen vor. Dadurch wirken sie einerseits auf eine Vielzahl von pharmakologischen Zielen und gehen in der Regel schwache Bindungen ein, d.h. sie bewirken keine vollständigen Blockaden/Stimulierungen/Sättigungen.

Das Wirkungsgefüge ist aufgrund vielfältiger Effekte und drogen- bzw. organismusbezogener Interaktionsmöglichkeiten funktionell $\mathrm{zu}$ betrachten. Durch Zusammenschau der Wirkung auf den verschiedenen Ebenen des Organismus lässt sich das Wirkprofil der traditionellen Anwendung erweitern, wobei für Arzneipflanzen die beiden polypharmakologischen Ansätze spezifisch sind:

- Multi-Target-Eigenschaften: Multiple, multifokale und multimodale Wirkungen. Multi-Target-Eigenschaften auf molekularer, genetischer und zellulärer Ebene sowie krankheitsbezogen und im Hinblick auf eventuelle Präventionsansätze. Viele solche partiellen Wirkeffekte können insgesamt wirksamer sein als z.B. die vollständige Hemmung eines «single targets», zudem scheinen solche Arzneimittel verträglicher zu sein. Analog zu den Auswirkungen der Multimorbidität auf den Kranken über verschiedene Netzwerkhierarchien des Organismus wirken solche Multi-Target-Drugs ebenfalls auf das Gesamtsystem.

- Pleiotrope Eigenschaften: Gleichzeitig mehrere bis zahlreiche, zum Teil voneinander unabhängige Wirkmechanismen. Daraus ergibt sich ein breites Wirkspek- trum, noch dazu mit relativ wenig Nebenwirkungen. Diese Eigenschaften ermöglichen eine ausgeprägte Plastizität, d.h. aufgrund seines vielschichtigen Wirkmechanismus kann sich das Vielstoffgemisch der dynamischen Multimodalität der Targets anpassen.

Die durch die experimentelle Forschung, insbesondere durch die Systembiologie aufgedeckten Wirkmechanismen erweitern die Erkenntnis über die systemische Wirkung der Arzneimittel.

\section{Pleiotrope Signaturen von Phytotherapeutika}

Stellt man die verschiedenen bekannten Wirkmechanismen und -orte einer Pflanze auf den unterschiedlichen Ebenen eines Organismus dar, z.B. Organsystem, Metabolismus, Proteinfunktion und Genexpression, so ergibt sich ein komplexes Wirkmuster. Versteht man den Organismus als ein hierarchisch geschichtetes Netzwerk, beeinflusst ein Multi-TargetArzneimittel das Gesamtsystem auf mehreren Hierarchiestufen. Damit ergibt sich eine Signatur des Pharmakons, die das Reaktionsbild über die verschiedenen Ebenen hinweg abbildet (Abb. 1). Phytotherapeutika sind empirisch gefundene Multi-Target-Drugs mit einer pleiotropen Wirksignatur. Sie können auf den unterschiedlichen Systemstufen des Netzwerks therapeutisch eingreifen, weshalb sie auch als Netzwerkarzneien bezeichnet werden. Durch den pleiotropen Charakter ist dieses Muster im Allgemeinen breiter als bei Monosubstanzen und auf viele, vor allem schwach ausgeprägte Verbindungen ausgelegt. Insbesondere bei pflanzlichen Kombinationen, die aus der Erfahrungsmedizin stammen, ist das Multi-Target-Konzept und damit Pleiotropie besonders ausgeprägt. Solche Kombinationspräparate können sich deshalb auch besonders als eine systemische Basistherapie eignen, z.B. kombiniert mit selektiven Arzneimitteln und/oder bei multimorbiden Patienten.

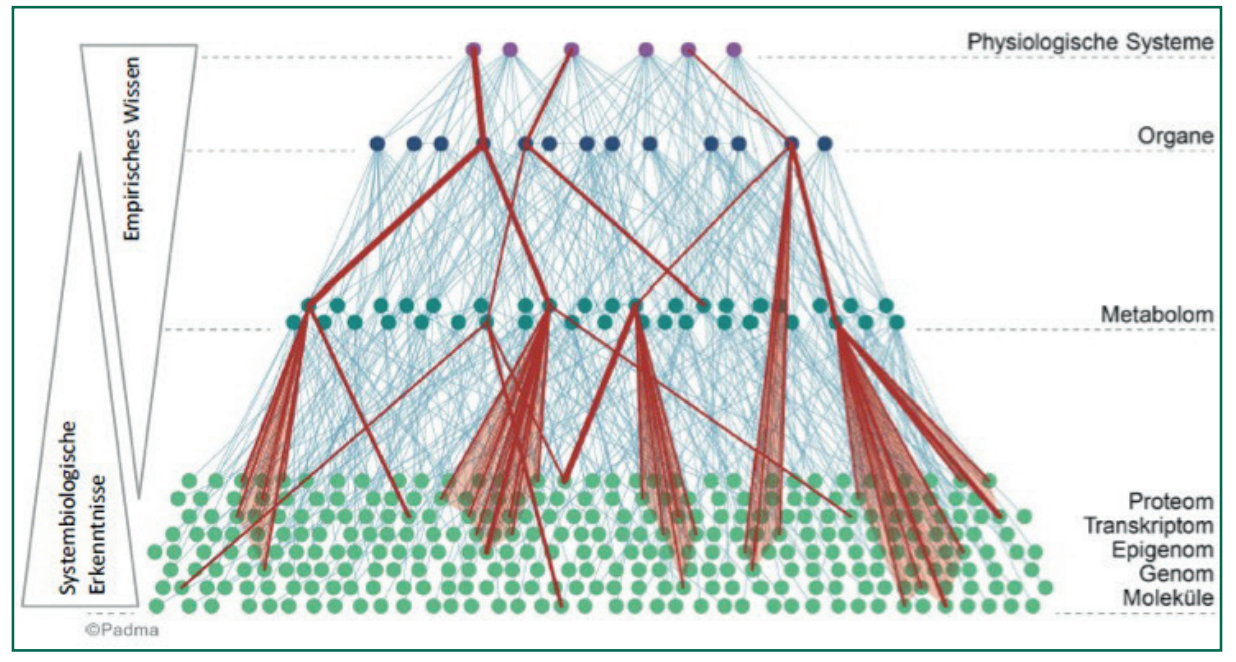

Abb. 1. Pleiotrope Wirksignatur eines Phytoarzneimittels in der Netzwerkhierarchie im menschlichen Organismus. Die Punkte symbolisieren die empirischen und experimentellen Daten der jeweiligen Hierarchiestufe. Blau eingezeichnet ist die Gesamtheit der möglichen Interaktionen. Rot hervorgehoben sind durch eine Interaktion mit dem System tatsächlich agesprochene Signalwege, die Dicke der Linie kennzeichnet die Verbindungsstärke. Dies bildet die charakteristische Signatur. Im Fall von Phytoarzneimitteln weiten sich die Signalwege durch den Multi-Target-Charakter aus (angedeutet durch die Schattierung auf den unteren Netzwerkstufen) - ein Charakteristikum der pleiotropen Signatur. 
Dies erweitert und ordnet das aus der überlieferten Empirie beschriebene Netzwerk der physiologischen Syteme, Organe und Gewebe. Der heute aktuelle Forschungsansatz der Systembiologie bezieht Systeme weiterer Hierarchiestufen in ein allgemeines Funktionsbild mit ein, von der Gen-TranskriptStufe über das Proteom, Metabolom und Epigenom hin zum Netzwerk der gesamten molekularen Interaktionen, dem sogenannten Interaktom. Die systembiologische Betrachtung komplettiert das empirische Netzwerk zu einer Gesamtschau der pleiotropen Signatur.

Es ist dabei wichtig zu erkennen, dass damit das überlieferte empirische Wissen sozusagen durch weitere Ebenen unterlegt wird. Es kommt also zu einer traditionellen Empirie mit systembiologischer Erweiterung: Neue Erkenntnisse kommen durch die neuen Methoden der Systembiologie zwar hinzu, das traditionelle Anwendungsgebiet bleibt jedoch erhalten (so wie ja auch schon beim antiken Menschen die genetischen Vorgänge durchaus vorhanden waren, obwohl das genetische Paradigma noch unbekannt war).

Die vollständige pleiotrope Signatur drückt damit das gesamte bereits in der Erfahrung angelegte therapeutische Potential eines Wirkstoffgemisches aus. Damit wird das traditionelle Wissen mit den neuen Erkenntnissen verknüpft, damit aufgewertet und frisch belebt.

\section{Traditionelle pflanzliche Arzneimittel: notwendig für die Zukunft}

Die therapeutische Anwendung pflanzlicher Arzneimittel erfolgt nach folgenden Hauptmotiven:

- Indikationsbezogen: Eingegrenzte Indikation als Teilaspekt bzw. Sonderfall des genuinen phytotherapeutischen Potentials. Die jeweiligen Indikationen sind ein Spezialfall des gesamten Indikationspotentials.

- Systemmittel: Ausnutzung von systemischen Begleitwirkungen, z.B. antiinflammatorischen Eigenschaften bei Krankheiten, bei denen chronische Entzündungsvorgänge pathogenetisch eine Rolle spielen.

- Komplexe Krankheitsbilder bzw. Therapiesituationen, z.B. Multimorbidität: Qualifizierte Ausnutzung der polypharmakologischen Wirkstoffeigenschaften und der zumeist guten Verträglichkeit (Polypharmakotherapie).

In der derzeitgen Praxis der Registrierung von traditionellen Arzneimitteln wird vor allem der klassische indikationsbezogene Zugang als adäquat betrachtet.

Durch die Darstellung als pleiotrope Signaturen lässt sich auch die Anwendung als Systemmittel oder bei komplexen
Therapiesituationen plausibel erklären. Diese Angaben finden sich ja ebenso in der überlieferten Tradition dieser Arzneimittel, werden aber oft als unspezifisch oder ungeeignet für die Indikationsangabe auf der Arzneispezialität angesehen.

Da die systembiologische Erweiterung die in der Literatur angegebenen Anwendunggebiete untermauert, wird die empirisch vorgegebene Basis (mit ihrer gesetzlich vorgegebenen Grenze von 30 Jahren) erweitert. Diese Erweiterung stellt nicht eine neue Indikation dar, sondern vielmehr einen wissenschaftlich herausgearbeiteten Teilaspekt eines prinzipiell umfassenden Wirkpotentials. Die Einbeziehung neuer Forschungsergebnisse stärkt den traditionellen Erfahrungsschatz und erweitert den Blick von der indikationsbezogenen Sicht hin zum Systemmittel und zur Anwendung in komplexen Therapiesituationen.

Die regulatorische Berücksichtigung der stets vorhandenen Möglichkeiten des systemischen Indikationsfeldes der pflanzlichen Zubereitung würde zweifellos die Attraktivität der Kategorie der traditionellen pflanzlichen Arzneimittel erhöhen - ein adäquater und innovativer Schritt, der für die wissenschaftliche Anwendbarkeit, Akzeptanz und den Erfolg dieser Arzneimittel notwendig ist. Schließlich geht es um das qualitätsbasierte Überleben einer intellektuell und therapeutisch attraktiven Arzneimittelkategorie.

\section{Literatur}

EC (European Commission): «Directive 2001/83/EC of the European parliament and of the Council of 6 November 2001 on the community code relating to Medicinal products for human use», EU Official Journal L-311, 28/11/2004, pp 67-128. Schwabl H, Vennos C, Saller R: Tibetische Rezepturen als pleiotrope Signaturen Einsatz von Netzwerk-Arzneien bei Multimorbidität. Forsch Komplementmed 2013;20(suppl 2):35-40.

\section{Disclosure Statement}

Jörg Melzer: JM erhielt keine Forschungsgelder für seinen Beitrag. Es besteht kein Interessenkonflikt.

Günter Meng: Interessenkonflikte: Keine. Der Autor ist Leiter Forschung und Entwicklung der Dr. Willmar Schwabe GmbH \& Co. KG, eines international tätigen Unternehmens, das sich mit der Entwicklung und dem Vertrieb pflanzlicher Arzneimittel beschäftigt.

Ursula von Mandach, Karin Fürer: Die Bryophillum Study Group wurde von Weleda AG in Arlesheim unterstützt.

Herbert Schwabl, Cecile Vennos, Reinhard Saller: Es bestehen keine Interessenkonflikte. HS und CV sind Mitarbeiter der Padma AG. 